\title{
Lotteries as Incentives in Longitudinal Web Studies
}

\author{
Anja S. Göritz \\ Hans-Georg Wolff \\ University of Erlangen-Nürnberg
}

\begin{abstract}
A longitudinal experiment examined the influence of a lottery of gift certificates on response and retention in a four-wave study conducted in an online panel. Independent of the lottery, people who responded in a given wave were more likely to respond in the next wave. This process was characterized to follow a first-order Markov chain. There was a direct positive effect of the lottery on response only at the first wave of the study. However, mediated by the Markov process, the positive effect of the lottery on response at the first wave was carried over into later waves. The lottery did not have any effect on retention. Furthermore, it was found that retention at a given wave is a reliable predictor for response at the next wave. This information could be used by survey managers to diagnose and act on any impending nonresponse.
\end{abstract}

Keywords: incentive; experiment; longitudinal; response; retention; online panel

\begin{abstract}
A $\mathrm{n}$ online panel is a pool of people who have signed up to occasionally take part in Web surveys. Online panels have become popular as platforms for data collection (Couper, 2005; Göritz, Reinhold, \& Batinic, 2002) because of their many benefits, such as easy identification of key sample segments, usually high response rates, short field times, validation of respondents' answers on the basis of previously collected data, limitation of questionnaires to novel items, and ethical advantages (e.g., Göritz, in press). Because in online panels respondents are asked to repeatedly participate in online studies, it is important to keep respondents committed in an ongoing fashion. The present study examines the effect of repeated lotteries on participation in a four-wave, longitudinal survey that was conducted in an online panel.

Despite Dillman's (2000) conclusion that lotteries have a "relatively small, if any, effect" (p. 169) on participation in offline surveys, lotteries have successfully been utilized in online surveys (e.g., Bošnjak \& Tuten, 2003). In fact, they are the most common type of incentive used in online studies (e.g., Göritz, 2006a; Göritz et al., 2002; Musch \& Reips, 2000; Tuten, Galešić, \& Bošnjak, 2004). Lotteries cause little transaction costs because only a few winners need to be sent a prize and costs are typically capped (i.e., unless every $n$th participant gets a prize, the costs stay the same regardless how many participants eventually take part). However, almost all studies that have examined the effectiveness of lotteries in Web-based studies (for a meta-analysis, see Göritz, 2006a) utilized cross-sectional designs. That is why little is known about the effect of lotteries in longitudinal studies. Results from cross-sectional studies may not generalize to longitudinal studies because
\end{abstract}

Authors' Note: This work was supported by a University of Erlangen-Nürnberg postdoctoral scholarship (HWP) to the first author. 
with longitudinal studies, participants repeatedly decide whether to participate in a given wave. Participants thus gain experience with the survey, which may affect their decision to participate in a subsequent wave (e.g., participants may remember whether they participated in previous waves or whether the study was interesting or tedious to complete, etc.; Hill \& Willis, 2001).

The four-wave study presented in this article examines the effectiveness of a lottery on study participation compared to a no-incentive control group in an online panel. With regard to study participation, two facets can be distinguished. The first facet is response, which refers to participants' calling up the first page of the study. The response rate is the ratio of people who load the first page of a study to all people who were invited to this study. The opposite of response is nonresponse (sometimes also called refusal). The second facet is retention. Retention indicates whether participants who have loaded the first page of the study stay until the last page of the study. The opposite of retention is dropout. Dropout causes incomplete data that often cannot be used in further analyses (Bošnjak \& Tuten, 2003; Porter \& Whitcomb, 2005). Although in paper-and-pencil studies retention is often muddled with response, in Web-based studies participants' answers are usually recorded in a page-by-page fashion, and therefore retention can be explicitly studied. Because different processes may underlie participants' decisions to respond to a survey request and — once having loaded the first study page — to stay until the last page, these two facets of study participation will be considered separately.

Offline studies (e.g., Lee, Hu, \& Toh, 2004) and online studies (Göritz, 2006b; Hiskey $\&$ Troop, 2002) show that response rates in longitudinal studies typically decrease over time, whereby only highly motivated participants remain committed at later survey waves. This longitudinal decline in response can be described by a Markov process (e.g., Hagenaars, 1990; Langeheine \& Van de Pol, 1990). Therefore, Hypothesis 1 postulates that our data can be described by a Markov process as well. A Markov process implies that those people who take part in a survey wave are more likely to take part again than are nonrespondents. In a first-order Markov process, the likelihood of responding at a given wave $w$ depends on response at the prior wave $w-1$. In a second-order Markov process, the likelihood of responding at a given wave depends on response at the two prior waves, $w-1$ and $w-2$, respectively. Supplementing Hypothesis 1, we will examine whether a first-order or higher-order Markov process best describes the longitudinal pattern of response.

According to theories on panel response (e.g., Hill \& Willis, 2001), participants weigh the costs (e.g., time) and benefits of responding (e.g., satisfaction of curiosity or altruistic motives) against each other. The offer to be included in a lottery and the ensuing chance of winning a prize is an additional benefit that should increase the response rate. In examining the effect of a lottery on response in a longitudinal setting, two alternative predictions can be derived that will be tested against each other. First, generalizing from cross-sectional studies (e.g., Tuten et al., 2004), a straightforward assumption is that the effect of the lottery on response is constant across survey waves. In contrast to a no-lottery situation, participants eligible for the lottery are offered a reward, and this additional benefit should result in higher response rates at all waves when such a lottery is offered. Thus, in Hypothesis $2 \mathrm{a}$, we predict a constant, positive effect of the lottery on response across survey waves. A second, alternative prediction (= Hypothesis $2 \mathrm{~b})$ can be derived from the 
Figure 1

Modeling the Effect of the Lottery on Response

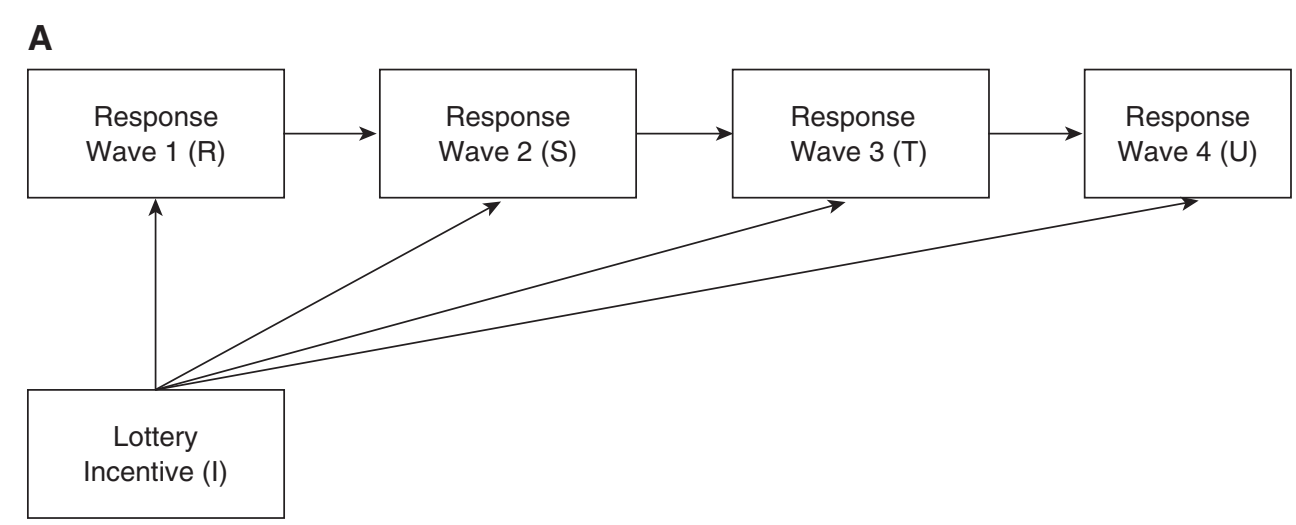

B

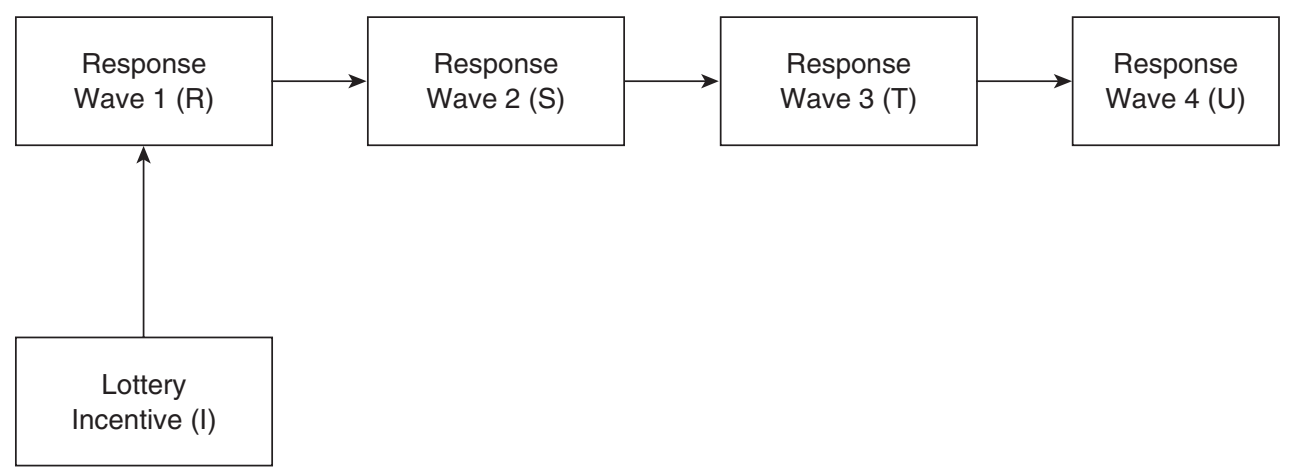

Note: Panel A = direct effect of the lottery on Waves 1 to 4 . Panel B = indirect effect of the lottery on Waves 2 to 4 .

fact that most participants who are included in the lottery drawing will not actually win anything. Similar to cross-sectional studies, participants may be initially motivated to respond by the possibility of winning a reward, and they may also overestimate the probability of winning (e.g., Brandstätter, Kühberger, \& Schneider, 2002). However, over time participants learn that they most likely will not win a reward and may hence readjust their cost-benefit calculations accordingly. Participants may also be disappointed because they did not receive the reward for which they had hoped. If this holds, the effect of a lottery on response should decline across consecutive waves of a longitudinal study. In comparison to the no-lottery control group, the response rate in the lottery group is higher in the beginning of a longitudinal study and will drop (perhaps even below the response rate of the control group) at later waves. Thus, Hypothesis $2 \mathrm{~b}$ predicts that the effect of the lottery on response declines over the survey waves. The model according to Hypotheses $2 \mathrm{a}$ and $2 \mathrm{~b}$ is depicted in Panel A of Figure 1. The lottery has an effect on reponse at each survey wave. The difference between the hypotheses lies in the restriction of these paths. 
According to the constant effect predicted in Hypothesis 2a, the paths are restricted to be equal, whereas according to Hypothesis $2 b$, the paths from the lottery to each survey wave are fixed to a linearly declining pattern (e.g., 3, 2, 1, and 0). Figure 1 also includes a Markov process as postulated in Hypothesis 1. In this case, a first-order Markov process is depicted, which is reflected by the effect of each survey wave on the subsequent wave.

From Panel A of Figure 1, it is evident that the lottery has direct effects on response at each wave but also an indirect effect that is mediated by the Markov process. The model presented in Panel A of Figure 1 thus assumes partial mediation. However, a full mediation model, as depicted in Panel B of Figure 1, is conceivable as well. In this model, there exists a direct effect of the lottery on response at Wave 1 but not in later waves. Yet differences in response at Wave 1 that were brought about by the lottery will be carried over onto subsequent waves by means of the Markov process. In line with the rules for examining mediation (Baron \& Kenny, 1986), if both Hypothesis 1 and Hypothesis $2 \mathrm{~b}$ are supported, we will also examine whether full mediation holds. Accordingly, in Hypothesis 3 we predict a direct effect of the lottery on response at Wave 1, yet at later survey waves, the lottery merely has indirect effects that are mediated by the Markov process predicted by Hypothesis 1 .

The offer of a lottery might not only increase participants' motivation to look at a survey; a lottery might also increase participants' retention. All other things being equal, the additional benefit incurred by the lottery may outweigh the costs of filling in a boring or tedious questionnaire with the result that with a lottery on offer, more people stay until the end of a study than would have done without a lottery. Even if such a conditionality is not spelled out explicitly, participants who are offered to be included in a lottery may implicitly assume that only those people who stay until the end of the questionnaire are entered into the lottery. Thus, Hypothesis 4a posits a constant, positive effect of the lottery on retention across survey waves.

Again, it is also conceivable that the effect of the lottery on retention declines across survey waves. This would imply that the lottery affects participants' motivation to complete the questionnaire, and because of growing experience, the lottery would over time lose its importance as a motivator for participants to stay until the last survey page. As an alternative to Hypothesis 4a, we therefore predict in Hypothesis $4 \mathrm{~b}$ that the effect of the lottery on retention declines across waves.

Beside analyzing the effect of the lottery on panelists' participation, the longitudinal design allows for analyzing whether dropout in an earlier survey wave is a predictor of nonresponse at a later wave (see Loosveldt, Pickery, \& Billiet, 2002; Serfling, 2005). This topic has never been examined in the context of Web-based longitudinal studies. Dropout at an earlier wave might be the result of a participant's flagging interest, or it might be the result of technical problems. Both reasons render it more likely that participants will abstain from responding to future survey requests. Accordingly, in Hypothesis 5 we assume that dropout at a given wave is a predictor of nonresponse at the subsequent wave. If the validity of dropout as an early indicator of impending nonresponse were confirmed, survey managers could take special action to prevent dropouts from failing to respond at the next survey wave. 


\section{Method}

In Wave 1, which was conducted from March 25 until April 1, 2003, 381 panelists from a German university-based online panel, who on their registration had declared to be employees, were invited to a study about relationships at work. A total of 161 women $(42.3 \%)$ and 220 men were invited. Their average age was 36 years $(S D=9)$. The e-mail invitation stated that in a few weeks invitees would be contacted again to take part in the second wave of this study. All people were offered a summary of the results. A randomly selected group of the sample was assigned to the lottery condition. Participants in this condition were told that five gift certificates issued by a well-known online media store, each worth 20 Euros, would be raffled among people who participate in both waves. The other group was not informed of this lottery. Wave 2 was conducted from May 12 until May 19, 2003. All people who had responded in Wave 1, except 2 panelists whose e-mail addresses had meanwhile become invalid, were invited to Wave $2(N=232)$. In the invitation, people who had been assigned to the lottery condition were reminded of the lottery. Wave 3 took place from April 30 until May 12, 2004. All people who had participated in Wave 1, minus 6 people whose e-mail addresses had meanwhile become invalid, were invited $(N=228)$. People were reminded that they had participated in a study about relationships at work and that the present study was a follow-up. Again, everybody was offered a result summary, and participants from the lottery condition were informed of a lottery of five gift certificates, each worth 20 Euros. Wave 4 took place from April 5 until April 18, 2005. All people who had participated in Wave 1, minus 27 people whose e-mail addresses had meanwhile become invalid, were invited $(N=207)$. There were 3 panelists who had quit their panel membership between Wave 3 and Wave 4 . For Wave 4, they were regarded as invited but as having refused participation. Again, invitees were reminded of their prior participation and asked to complete this follow-up survey. All participants were offered a result summary, and participants from the lottery condition were informed of a lottery of five gift certificates, each worth 20 Euros. In neither wave was a reminder sent to people who failed to answer.

To analyze the data, we used log-linear path models that can be employed to capture the causal structure among several categorical variables (Hagenaars, 1990; Vermunt, 1997). These models allow the simultaneous consideration of response and retention in a single model and have been frequently used to model the Markov processes predicted in Hypothesis 1 (e.g., Langeheine \& Van de Pol, 1990). In addition, log-linear path models can handle missing data that occur in the present analyses because nonrespondents have missing values in the retention variable. We used an approach by Fay (1986; see also Hagenaars, 1990; Vermunt, 1997) to explicitly incorporate effects of nonresponse. In this approach, subgroups with different patterns of missing data are created and jointly analyzed by using the maximum amount of information available from these subtables. As our hypotheses refer to specific paths in the model, we tested several models in a stepwise manner (Hagenaars, 1990), successively adding and deleting hypothesized paths if this significantly improved model fit. We used conditional log likelihood ratio tests and the Bayesian information criterion (BIC) to evaluate different models (Vermunt, 1997). 
Table 1

The Effect of the Lottery on Response, Retention, and Usable Data in Waves 1 to 4

\begin{tabular}{|c|c|c|c|c|c|}
\hline & \multicolumn{2}{|c|}{ Lottery } & \multicolumn{2}{|c|}{ No Incentive } & \multirow{2}{*}{$\begin{array}{c}\text { Lottery Effect } \\
\%\end{array}$} \\
\hline & $n$ & $\%$ & $n$ & $\%$ & \\
\hline \multicolumn{6}{|l|}{ Response } \\
\hline Wave 1 & 130 of 192 & 67.7 & 104 of 189 & 55.0 & 12.7 \\
\hline Wave 2 & 106 of 130 & 81.5 & 75 of 102 & 73.5 & 8.0 \\
\hline Wave 3 & 71 of 127 & 55.9 & 63 of 101 & 62.7 & -6.8 \\
\hline Wave 4 & 62 of 116 & 53.4 & 52 of 91 & 57.1 & -3.7 \\
\hline \multicolumn{6}{|l|}{ Retention } \\
\hline Wave 1 & 123 of 130 & 94.6 & 98 of 104 & 94.2 & 0.4 \\
\hline Wave 2 & 95 of 106 & 89.6 & 70 of 75 & 93.3 & -3.7 \\
\hline Wave 3 & 65 of 71 & 91.5 & 50 of 63 & 79.4 & 12.1 \\
\hline Wave 4 & 54 of 62 & 87.1 & 44 of 52 & 84.6 & 2.5 \\
\hline \multicolumn{6}{|l|}{ Usable data } \\
\hline Wave 1 & 123 of 192 & 64.1 & 98 of 189 & 51.9 & 12.2 \\
\hline Wave 2 & 95 of 130 & 73.1 & 70 of 102 & 68.6 & 4.5 \\
\hline Wave 3 & 65 of 127 & 51.2 & 50 of 101 & 49.5 & 1.7 \\
\hline Wave 4 & 54 of 116 & 46.6 & 44 of 91 & 48.4 & -1.8 \\
\hline
\end{tabular}

\section{Results}

Independent of the incentive, the response rate of $61.4 \%$ in Wave 1 rose to $78.0 \%$ in Wave 2, which is a result of inviting to Wave 2 only those people who had responded to Wave 1 (see Table 1). Throughout the remainder of the longitudinal study, the response rate fell to $58.8 \%$ in Wave 3 and to $55.1 \%$ in Wave 4 . Table 1 lists the response and retention rates for the lottery and control conditions and the net effect of the lottery. The net effect of the lottery on response declines from a positive value of $12.7 \%$ to a negative value of $-2.7 \%$. Net effects of the lottery on retention do not yield a systematic pattern over time. Table 1 also shows the total effects of the lottery by combining the effects on response and retention (= useable data). The total effect of the lottery declines from a positive value of $12.2 \%$ to $-1.8 \%$

To test Hypotheses 1 to 5, we tested 10 models that are depicted in Table 2. Response variables for Waves 1 to 4 are labeled $R, S, T$, and $U$, respectively. Similarly, retention variables for the four waves are labeled $A, B, C$, and $D$. The lottery variable is labeled $I$. Models are labeled by their sufficient marginals (see Hagenaars, 1990); for example, IA denotes an effect of the lottery $(I)$ on retention at Wave $1(A)$. Hypotheses tests proceeded in four steps, examining different parts of the model. In each step, the best fitting model from the prior step (i.e., the model that included significant hypothesized paths and dropped unconfirmed hypothesized paths) was used as a baseline model. Also, a saturated model was used for the retention process in Steps 1 and 2 because these two steps focused on response variables only (i.e., all possible main effects and interaction terms of retention were included in the model; Vermunt, 1997). 


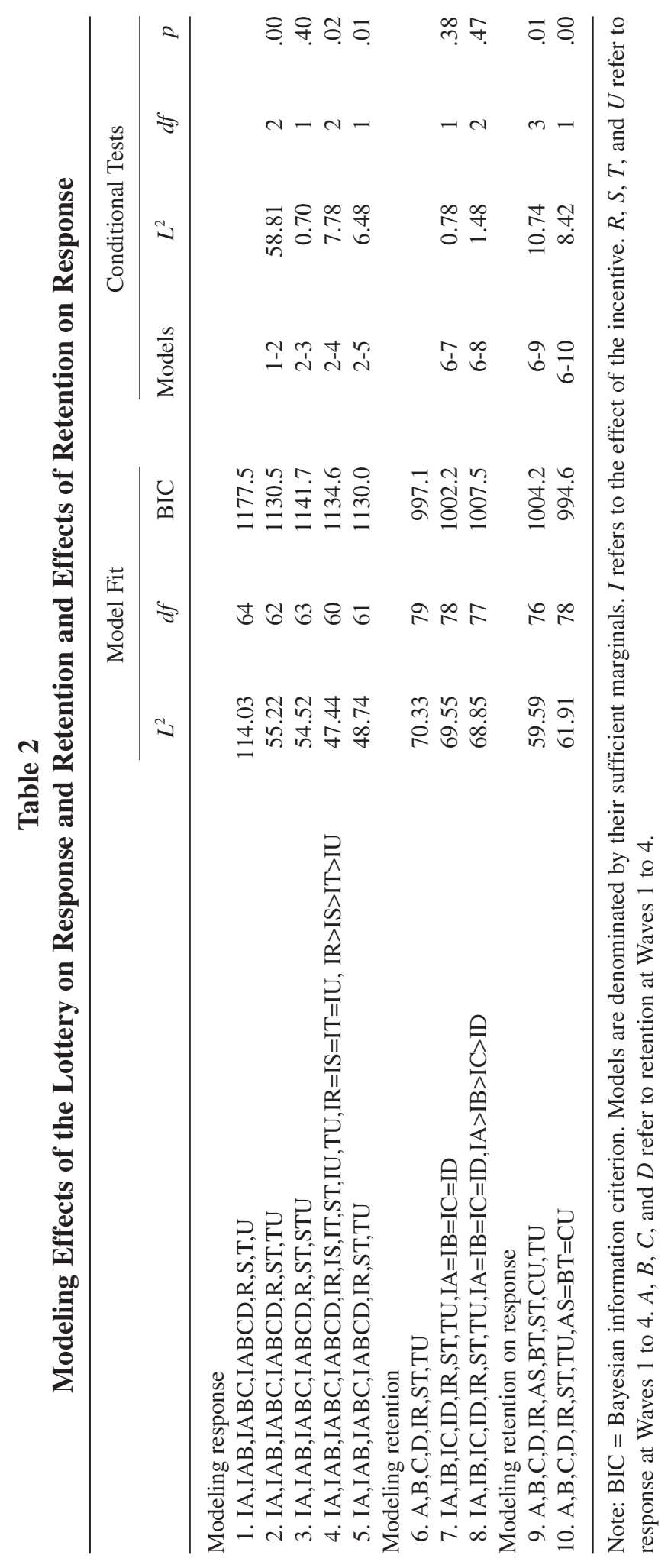


In Step 1, we examined whether response variables are related across waves by a Markov process, as predicted in Hypothesis 1. Model 1 serves as a baseline model and assumes the absence of a Markov process; that is, it assumes independence among response variables at different waves. Model 2 reflects a first-order Markov process where response at a given wave depends on response at the previous wave. This model fits significantly better than Model 1 (see conditional test of Model 1 versus Model 2 in Table 2). Model 3 represents a second-order Markov process, but in comparison to Model 2, Model 3 does not further improve fit. Thus, a first-order Markov process best describes the dependency among response variables at consecutive waves. Hypothesis 1 receives support.

In Step 2, the lottery effect on response is examined in accordance with Hypotheses 2 and 3, using Model 2 as a baseline model. For reasons of brevity, we do not report tests for Hypothesis 2a that predicted a constant, positive effect of the lottery. From Table 1, the absence of a constant effect is evident. Thus, Hypothesis $2 \mathrm{a}$ is rejected. Model 4 in Table 2 tests the linearly declining effect of the lottery as predicted by Hypothesis $2 b$. The decline is indicated by equality and inequality constraints on effects $I R, I S, I T$, and $I U$. This model has two coefficients; one coefficient reflects the effect of the lottery on response at Wave 1, and the other coefficient reflects this effect's change over time. Model 4 fits significantly better than Model 2. The coefficient for the effect of the lottery in Wave 1 indicates that the lottery significantly increases response $(b=0.54, z=2.77)$. The coefficient for the change over time indicates that the effect of the lottery declines linearly over time $(b=-0.24, z=2.21)$. According to this model, the odds ratio at Wave 1 is 1.71 , indicating a difference in response of $13 \%$ between the lottery condition $(68 \%)$ and the no-lottery condition $(55 \%)$. Over time, the odds ratio decreases to 0.80 at Wave 4, indicating that in Wave 4, people in the lottery condition are less likely to respond than people in the control condition. This corresponds to a $4 \%$ difference in favor of the control condition. Thus, Hypothesis $2 \mathrm{~b}$ receives support.

Because Hypothesis 1 (assuming a Markov process) and Hypothesis $2 \mathrm{~b}$ (assuming a decline of the lottery effect) were supported, we tested Hypothesis 3, which postulates an indirect effect of the lottery on response at Waves 2 to 4. In comparison to Model 4, Model 5 contains only the indirect effect as depicted in Panel B of Figure 1. This model fits significantly better than Model 2, which did not contain a lottery effect. Therefore, Hypothesis 3 is supported, which posits a direct effect of the lottery on response at Wave 1 and indirect effects in subsequent waves.

Next, we modeled the retention part of the model. With regard to the response part of the model, Model 5 will be used as a baseline model. As we did not expect a specific model for the relationship between retention variables across waves, we explored several possible models whose results will not be reported here for brevity. The best fitting model postulates independence between retention variables at the four survey waves and is depicted as Model 6. To test Hypotheses $4 \mathrm{a}$ and $4 \mathrm{~b}$, we entered effects of the lottery on retention. Model 7 includes a constant effect of the incentive on retention as postulated in Hypothesis 4a. Because Model 7 does not improve fit compared to Model 6, Hypothesis 4a is rejected. Next, we tested a declining effect of the lottery on retention as postulated in Hypothesis $4 \mathrm{~b}$ (Model 8), but including this effect into the model did not improve model fit, either. Therefore, Hypothesis $4 \mathrm{~b}$ is rejected.

Finally, to test Hypothesis 5, we added effects of retention at the given wave on response at the next wave, resulting in Model 9 (e.g., effect $A S$ in Table 2 indicates that retention at 
Figure 2

Final Model for This Study's Data Set

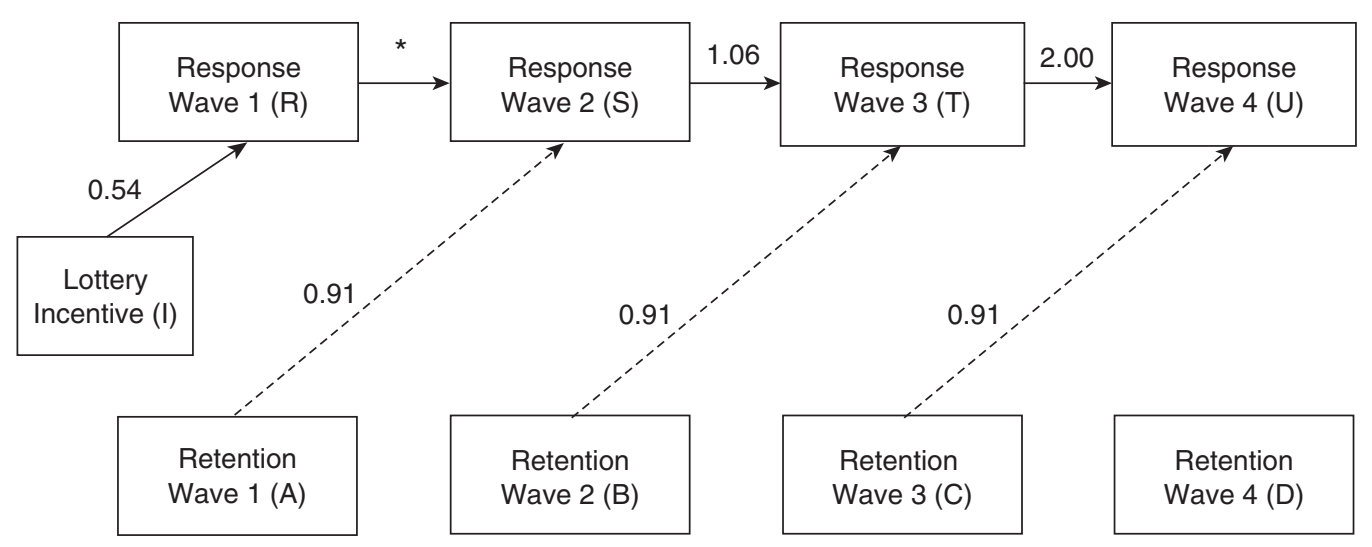

Note: Variable names in accordance with Table 2 are included in parentheses. Log-linear coefficients are shown. Dotted lines indicate that these effects are restricted to be equal. The asterisk indicates a fixed path because of invitation procedures.

Wave 1 affects response at Wave 2). As we did not find any effects of the lottery on retention, Model 6 was used as the baseline model. Model 9 improves fit significantly. Coefficients indicate that respondents who drop out at the given wave are less likely to respond to the next wave than are respondents who do not drop out at the given wave. In an attempt to render the model more parsimonious, we restricted this effect to be equal across survey waves (i.e., we included constant effects of retention at a given wave on response at the next wave). The resulting Model 10 fits significantly better than Model 6 . Moreover, the BIC indicates that Model 10 also fits better than Model 9. Again, the effect of retention on response indicates that participants who drop out at a given wave are less likely to respond at the next wave $(b=-0.91, z=2.93)$. Thus, Hypothesis 5 is supported.

Figure 2 illustrates Model 10, which was the best model to account for the data set as a whole: Response at a given wave is affected by response and retention at the prior wave. The lottery promotes response at Wave 1 and has an indirect, positive effect on response at later waves, which is mediated by the first-order Markov process of response. The lottery does not have any effects on retention.

\section{Discussion}

Our study reveals that a lottery of gift certificates increases response in longitudinal online panel studies. We found a positive effect of the lottery on response at the first wave of the study but no further direct effects of the lottery at later waves. However, the lottery had an indirect effect on response at later waves: Because responding at a given wave makes it more likely to respond at the next wave, the positive effect of the lottery on 
response at the first wave is carried into later waves, which can be described by means of a first-order Markov process. In accordance with cost-benefit models of response (Hill \& Willis, 2001), we find that over time participants gain experience with the lottery, learning that the chance of actually winning a prize is low. Participants readjust their cost-benefit calculation in accordance with their experience when deciding whether to respond at the next opportunity.

To provide an account of the magnitude of the lottery effect on response, consider the following scenarios: In Scenario A, a lottery of five gift certificates, each worth 20 Euros, is offered as an incentive at every survey wave. In Scenario B, everything else is identical except that no incentive is offered. In each scenario, we invite 1,000 people to Wave 1, and we carry out a total of four survey waves. Relying on the findings of the present study, we would end up with 315 usable data sets in Scenario A and 266 in Scenario B. At the fourth wave, the size of the remaining sample in Scenario A is about $18 \%$ (49 people) higher than in Scenario B. This gain in sample size would have been bought with 400 Euros incentive money, which is roughly 8 Euros per respondent. This figure may orient readers to the order of magnitude of expectable effects. Within their particular study context (e.g., the aims of the study, the nature of the sample, the sample size), they can judge whether this money is well spent.

In contrast to response-as one facet of study participation-we found no effect of the lottery on retention - as another facet of study participation. Any impact of a lottery becomes fully absorbed by the response variable. In other words, participants' expectations and disappointments in connection with the lottery affect their decision to respond but not their decision to drop out of the study. This null effect of a lottery on retention may be specific to online panels, as it is in line with other findings stemming from online panel studies (Göritz, 2006c) but stands in contrast to positive effects of a lottery on retention from studies conducted outside of online panels (Bošnjak \& Tuten, 2003; Frick, Bächtiger, \& Reips, 2001; O’Neil, Penrod, \& Bornstein, 2003; Tuten et al., 2004). In any case, the noneffects of a lottery on retention in this study cannot be interpreted as proof that no such effects exist. With the small number of dropouts (e.g., fewer than 10 in most conditions, see Table 1), the overall variability of retention in this study might have been insufficient to detect any effects of the lottery on retention. More studies are needed to resolve the hitherto inconclusive findings on the effect of a lottery on retention.

Moreover, the study has demonstrated that in Web-based longitudinal studies, dropout in the present wave is a precursor of nonresponse in the following wave. Therefore, dropouts can be considered at risk of nonresponding, and measures to counteract this risk may be taken by the survey administrator. For example, to prevent subsequent nonresponse, dropouts can be contacted and offered technical support, or, in the case of participation fatigue, one may attempt to win them back by a refusal conversion incentive (Singer, 2002).

Our study is not without limitations. Our reliance on a nonprobability sample of online panel members may weaken the generalizability of the results. In addition, generalizability of the findings to studies using more than four waves or different intervals between waves remains to be established in future research. In the present study with four waves, the lottery was beneficial in terms of the percentage of people from the initial sample responding in the final wave. However, the advantage brought about by the lottery kept shrinking with each wave. If this declining trend-instead of plateauing - continued beyond four waves, at a 
particular number of waves higher than four, the use of lotteries would become counterproductive. This supposition can be tested in online panel studies with more than four waves.

Moreover, the present study is limited to the comparison between a lottery and a noincentive control group and thus cannot evaluate the effectiveness of the lottery in comparison with other types of incentives. Further research is needed to fill this gap. Also, future research may examine whether the carryover effect of the lottery from Wave 1 into subsequent waves holds even if a lottery was offered at Wave 1 only, instead of being offered at each wave, as was done in the present study. A strength of our study is the random assignment of participants to experimental conditions, which renders alternative explanations for the lottery effect unlikely. We also believe that the observation of panel members during a 2 -year period is a unique contribution of the present study.

In sum, our results show that lotteries reduce nonresponse in longitudinal studies conducted in online panels. In conjunction with their easy implementation, lotteries can be considered a viable and cost-efficient tool in longitudinal studies_at least in those consisting of up to four waves.

\section{References}

Baron, R. M., \& Kenny, D. A. (1986). The moderator-mediator variable distinction in social psychological research: Conceptual, strategic, and statistical considerations. Journal of Personality and Social Psychology, 51, 1173-1182.

Bošnjak, M., \& Tuten, T. L. (2003). Prepaid and promised incentives in Web surveys. Social Science Computer Review, 21, 208-217.

Brandstätter, E., Kühberger, A., \& Schneider, F. (2002). A cognitive-emotional account of the shape of probability weighting function. Journal of Behavioral Decision Making, 15, 79-100.

Couper, M. P. (2005). Technology trends in survey data collection. Social Science Computer Review, 23, $486-501$.

Dillman, D. A. (2000). Mail and Internet surveys. New York: John Wiley.

Fay, R. E. (1986). Causal models for patterns of nonresponse. Journal of the American Statistical Association, 81, 354-365.

Frick, A., Bächtiger, M.-T., \& Reips, U.-D. (2001). Financial incentives, personal information and drop-out in online studies. In U.-D. Reips \& M. Bošnjak (Eds.), Dimensions of Internet science (pp. 209-219). Lengerich, Germany: Pabst.

Göritz, A. S. (in press). Using online panels in psychological research. In A. Joinson, K. McKenna, T. Postmes, \& U.-D. Reips (Eds.), Oxford handbook of Internet psychology. Oxford, UK: Oxford University Press.

Göritz, A. S. (2006a). Incentives in Web studies: Methodological issues and a review. International Journal of Internet Science, 1(1), 58-70.

Göritz, A. S. (2006b). The long-term effect of material incentives on participation in online panels. Manuscript submitted for publication.

Göritz, A. S. (2006c). Cash lotteries as incentives in online panels. Social Science Computer Review, 24 (4), pp.XXX-XXX.

Göritz, A. S., Reinhold, N., \& Batinic, B. (2002). Online panels. In B. Batinic, U.-D. Reips, \& M. Bošnjak (Eds.), Online social sciences (pp. 27-47). Seattle, WA: Hogrefe \& Huber.

Hagenaars, J. A. (1990). Categorical longitudinal data. Newbury Park, CA: Sage.

Hill, D. H., \& Willis, R. J. (2001). Reducing panel attrition. Journal of Human Resources, 36, 417-438.

Hiskey, S., \& Troop, N. A. (2002). Online longitudinal research. Viability and participation. Social Science Computer Review, 20, 250-259.

Langeheine, R., \& Van de Pol, F. (1990). A unifying framework for Markov modeling in discrete space and discrete time. Sociological Methods and Research, 18, 416-441. 
Lee, E., Hu, M. Y., \& Toh, R. S. (2004). Respondent non-cooperation in surveys and diaries: An analysis of item non-response and panel attrition. International Journal of Market Research, 46, 311-326.

Loosveldt, G., Pickery, J., \& Billiet, J. (2002). Item nonresponse as a predictor of unit nonresponse in a panel survey. Journal of Official Statistics, 18, 545-557.

Musch, J., \& Reips, U.-D. (2000). A brief history of Web experimenting. In M. H. Birnbaum (Ed.), Psychological experiments on the Internet (pp. 61-87). New York: Academic Press.

O’Neil, K. M., Penrod, S. D., \& Bornstein, B. H. (2003). Webbased research: Methodological variables' effects on dropout and sample characteristics. Behavior Research Methods, Instruments, \& Computers, 35, 217-236.

Porter, S. R., \& Whitcomb, M. E. (2005). E-mail subject lines and their effect on Web survey viewing and response. Social Science Computer Review, 23, 380-387.

Serfling, O. (2005). The interaction between item, questionnaire and unit nonresponse in the German SOEP, Schmollers Jahrbuch-Journal of Applied Social Science Studies, 125 (1), 195-205.

Singer, E. (2002). The use of incentives to reduce nonresponse in household surveys. In R. M. Groves, D. A. Dillman, J. L. Eltinge, \& R. J. A. Little (Eds.), Survey nonresponse (pp. 163-177). Chichester, UK: Wiley.

Tuten, T. L., Galešić, M., \& Bošnjak, M. (2004). Effects of immediate versus delayed notification of prize draw results on response behavior in Web surveys-An experiment. Social Science Computer Review, 22, 377-384.

Vermunt, J. K. (1997). Log-linear models for event histories. Thousand Oaks, CA: Sage.

Anja S. Göritz is a postdoctoral fellow in the Department of Psychology at the University of Erlangen-Nürnberg, Germany. She may be reached at anja.goeritz@wiso.uni-erlangen.de.

Hans-Georg Wolff is a postdoctoral fellow in the Department of Psychology at the University of ErlangenNürnberg, Germany. He may be reached at hans-georg.wolff@wiso.uni-erlangen. de. 